\title{
Plataforma para el registro de nefrolitotomía percutánea interinstitucional
}

\section{Platform for the registration of inter-institutional percutaneous nephrolithotomy}

\author{
Catalina Solano Mendoza, MD ${ }^{1}$ Alfredo Ortiz, MD ${ }^{2}$ Adolfo Serrano, MD ${ }^{3}$ Raúl Rueda, MD
}

Nick Tarazona, MD ${ }^{5}$

${ }^{1}$ Urología, Uroclin, Medellín Antioquia, Medellín, Colombia

Address for correspondence Catalina Solano Mendoza, MD, Urología,

2 Dep. Urología, Centro urológico Foscal, Bucaramanga, Uroclin, Medellín Antioquia, Medellín, Colombia Santander, Colombia (e-mail: Catasolano84@yahoo.com).

${ }^{3}$ Dep. Urología, Fundación Santa Fe, Bogotá, Cundinamarca, Colombia

${ }^{4}$ Dep. Urología, Clínica Chicamocha, Bucaramanga, Santander, Colombia

${ }^{5}$ Dep Innovación Fundacionisa, Bogotá, Cundinamarca, Colombia

Urol Colomb 2018;27:147-150.

\section{Resumen}

\section{Palabras Clave}

- nefrolitotomía

- medicina basada en evidencia

- motor de búsqueda

- registro; Residentes

- urólogos

- endourología
Introducción Las opciones para el tratamiento de cálculos renales son: la litotripcia extracorpórea, la ureterorrenoscopia flexible y la nefrolitotomía percutánea (NLP). Se ha visto que a pesar del advenimiento de nuevas tecnologías y técnicas quirúrgicas, la NLP sigue presentando una importante tasa de complicaciones, por lo que es necesario la estandarización de la técnica y la escogencia adecuada de los pacientes.

Metodología Se realizó una búsqueda de la literatura en Pubmed sin límite de fecha en la que se tuviera en cuenta palabras claves como: registro de NLP, sistemas de puntuación en NLP, características preoperatorias tenidas en cuenta en NLP. Posteriormente se socializaron dichas variables con expertos en el tema, creando una plataforma que permitiera el registro de los pacientes a quienes se les realizó NLP en el país.

Resultados Se desarrolló una plataforma web para el registro a través de dispositivos móviles, tabletas, celulares, etc. de todos los pacientes a quienes se les realizó NLP en instituciones como: FOSCAL, Fundación Santa Fé de Bogotá y Clínica Chicamocha. Una vez ingresado los datos, estos se enviaron automáticamente a una base de datos consignados en una hoja tipo Excel. Por último se creó una red de especialistas para la participación virtual por medio de la misma.

Conclusiones El registro nacional de NLP es un estudio en tiempo real y de fácil acceso que permite obtener datos relevantes de los pacientes a quienes se les realiza NLP en varias instituciones del país. Adicionalmente proporcionará bases para la creación de estudios multicéntricos y publicaciones de literatura colombiana. received

November 29, 2016

accepted

April 16, 2017

published online

January 10, 2018
DOI https://doi.org/

10.1016/j.uroco.2017.04.007.

ISSN 0120-789X.
Copyright ( $)$ 2018, Sociedad Colombiana License terms de Urología. Publicado por Thieme Revinter Publicações Ltda., Rio de Janeiro, Brazil. Todos los derechos reservados. 


\begin{abstract}

\section{Keywords}

- nephrolithotomy

- evidence-based medicine

- search engine

- registry

- medical residents

- urologists

- endourology

Introduction The options for the treatment of renal calculi include extracorporeal lithotripsy, flexible ureterorenoscopy, and percutaneous nephrolithotomy (PNL). Despite the advent of new technologies and surgical techniques, it has been shown that PNL continues to present an important rate of complications, highlighting the need to standardise the technique and the appropriate choice of patients.

Methodology A search of the literature was carried out, with no date limits, using key words such as: PNL record, PNL scoring systems, and preoperative characteristics taken into account in PNL. These variables were then discussed with experts on the subject. This led to the creation of a platform that would allow the registration of the patients in whom PNL was performed in the country.

Results A web platform was developed for the registration, using mobile devices, tablets, mobile phones, etc., of all the patients in whom PNL was performed in institutions such as: FOSCAL, Fundación Santa Fe de Bogotá, and Clínica Chicamocha. Once the details were entered, they were sent automatically to a database entered in an Excel sheet. Finally, a network of specialists was created for virtual participation. Conclusions The national PNL registry is a real-time, easily accessible study that allows obtaining relevant data from the patients on whom PNL is performed in several institutions of this country. It will also provide bases for the creation of multicentre studies and publications from the Colombian literature.
\end{abstract}

\section{Introducción}

Desde la primera introducción de la nefrolitotomía percutánea (NLP) por Fernstrom y su popularización en EE. UU. por Smith, la NLP se ha convertido en el estándar de oro para pacientes con cálculos renales de gran tamaño. ${ }^{1}$

Las opciones actuales para el tratamiento de cálculos renales son: la litotripcia extracorpórea, la ureterorrenoscopia flexible y la NLP, escogiendo cada una según el tamaño del cálculo, la anatomía del sistema colector, las características del paciente y las preferencias del especialista. ${ }^{2}$ Para cálculos piélicos o de cáliz superior mayores de $2 \mathrm{~cm}$ o para cáliz inferior mayores de $1,5 \mathrm{~cm}$, se prefiere la NLP. ${ }^{2}$

Se ha visto que a pesar del advenimiento de nuevas tecnologías y técnicas quirúrgicas, la NLP sigue presentando una importante tasa de complicaciones, por lo que es necesario la estandarización de la técnica y la escogencia adecuada de los pacientes.

Desde el 2011 el sistema de puntuación de Guy (GSS), nefrolitometría S.T.O.N.E y el normograma de la clínica de investigación de la Sociedad de Endourología (CROES, por sus siglas en inglés), se han propuesto como un medio de evaluación que ayuda en la escogencia de la técnica, teniendo en cuenta las características preoperatorias del cálculo y el paciente. ${ }^{3,4}$

El objetivo de este estudio es crear una herramienta web que tenga las principales variables involucradas en estos 3 sistemas de puntuación y que permitan el registro de la NLP de todas las instituciones del país donde se realiza dicha técnica; adicionalmente el objetivo también es que exista un espacio de comunicación virtual con todos los urólogos del país inscritos, para la discusión de casos clínicos, y cuenta con las principales revista de endourología para tener acceso a literatura actualizada.

\section{Materiales y métodos}

Se realizó una búsqueda de la literatura en la base de datos de Pubmed, sin límite de fecha con términos MeSH y palabras claves que involucraron: nefrolitotomía percutánea, registro de nefrolitotomía percutánea, sistemas de puntuación en NLP y características preoperatorias tenidas en cuenta en NLP; encontrándose 21 artículos con tópicos en común, como los son las escalas de puntuación GSS, S.T.O.N.E nephrolithometry, normograma CROES, dentro de los cuales se identificaron las variables que más se repetían y que habían sido socializadas y definidas por un panel de 85 expertos. ${ }^{5}$ Posteriormente fueron evaluadas por urólogos colombianos líderes de opinión claves en el tema, creando una plataforma que involucrara las principales características y que permitiera el registro a través de dispositivos móviles conectados a Internet (tabletas, celulares, computadores) de los pacientes a quienes se les realiza NLP, iniciando en 3 instituciones del país.

\section{Resultados}

Se desarrolló una plataforma web para el registro a través de dispositivos móviles, tabletas, celulares y computadores con conexión a Internet de todos los pacientes a quienes se les realiza NLP en instituciones como: FOSCAL, Fundación Santa Fe de Bogotá y Clínica Chicamocha.

Para el acceso a la misma, se ingresa a través del link nlp. fundacionisa.org, en donde aparecen varias opciones, dentro de las cuales se encuentran las principales revistas de 
endourología para la búsqueda de medicina basada en la evidencia relacionada con urolitiasis, también la opción de la comunicación en red con todos los urólogos inscritos, con el objetivo de discusión de casos clínicos, y por último la opción de estadística, en la cual se encuentran todas las variables a tener en cuenta para la creación del registro.

Variables demográficas como identificación, género, edad, peso; variables prequirúrgicas como lateralidad, tamaño del cálculo, localización, densidad del cálculo, número de cálculos y por último características quirúrgicas como localización de la punción, número de punciones, instrumentos de litofragmentación, tasa libre de cálculos, necesidad de nefrostomía o catéter doble j, tiempo quirúrgico, complicaciones tipo Clavien, cálculo único en cáliz superior o múltiples cálculos en anatomía usual o cálculo único con anatomía anormal; grado III, múltiples cálculos en anatomía anormal o cálculos en un divertículos caliciales o cálculo coraliforme parcial; grado Iv, cálculo coraliforme o cualquier cálculos en paciente con espina bífida o trauma raquimedular. ${ }^{6}$

Por último el normograma de CROES, el cual determina la posición del paciente (prono o supino), accesos(radiólogo o urólogo) localización de la punción (por encima de la $12 .^{\mathrm{a}}$ costilla, por debajo de la $11 .^{\mathrm{a}}$ o $12 .^{\mathrm{a}}$ costilla), técnica de dilatación del trayecto (balón o dilatadores), instrumentos de litofragmentación (neumático, ultrasónico, combinado, láser, electrohidráulico), tasa libre de cálculos, procedimiento secundario, complicaciones (sangrado, perforación pelvis renal, hidrotórax, falla para completar el procedimiento, fiebre, transfusión sanguínea) ${ }^{?}$

Hay varias diferencias importantes entre los sistemas de puntuación analizados actualmente. Considerando que la puntuación de S.T.O.N.E. se basa totalmente en los datos que se pueden obtener a partir de la tomografía preoperatoria, el GSS y la puntuación de CROES incluyen variables propias del paciente. El GSS sin embargo no incluye el tamaño del cálculo, el cual es un fuerte predictor de éxito. Todos los sistemas de puntuación incluyen una medida de la complejidad del cálculo, si es coraliforme completo o incompleto. 3,4

Aunque el nomograma CROES incluye la mayor parte de las variables que determinan la tasa libre de cálculos, es un sistema de puntuación complejo lo que limita su aplicación en la práctica diaria.

Actualmente, GSS, S.T.O.N.E. y el nomograma CROES representan las 3 herramientas de pronóstico más comúnmente usadas para la NLP. Aunque estos sistemas se construyeron de forma independiente y a través de diferentes metodologías, todos ellos son propuestos para ayudar al cirujano en la evaluación de complejidad del caso con el fin de predecir la tasa libre de cálculos y los riesgos de complicaciones. ${ }^{3,4}$

En los últimos años, la importancia de la presentación sistemática y estandarizada de los resultados después de varios procedimientos urológicos, incluyendo NLP, ha generado importancia. ${ }^{5,8,9}$ Aunque Hyams et al. habían destacado anteriormente una gran heterogeneidad en la presentación de informes de variables preoperatorias en el manejo quirúrgico de los cálculos renales, recomendaciones de consenso sobre normalización de los informes de los datos preoperatorios aún no se han propuesto. ${ }^{10}$ Por lo que herramientas de pronósticos preoperatorios pueden ser útiles no solo para estratificar a los pacientes en diferentes grupos de riesgo, sino también como un medio de normalización de los informes de datos de cohortes preoperatorios.

La medición de los resultados óptimos y la presentación de informes son la piedra angular de una buena investigación quirúrgica. Ambos, tanto como los resultados de eficacia y seguridad, deben ser medidos adecuadamente para permitir una evaluación fiable de calidad de la atención quirúrgica, así como para facilitar la comparación de los estudios clínicos con el objetivo de crear evidencia clínica. ${ }^{11}$

En nuestro medio, no existe literatura acerca de los resultados obtenidos en NLP. Se cree que para que esto sea posible es necesario que los investigadores y participantes reporten una información real acerca de los procedimientos. Por ello es de importancia adoptar un sistema único de clasificación de complejidad del cálculo y características del paciente, que facilitarán determinar el riesgo de la NLP.

Por este motivo se creó una herramienta que permitiera el registro en tiempo real de dichas variables, con el objetivo de estandarizar la técnica y obtener datos que permitieran la realización de trabajos de investigación, ya que hasta la fecha en nuestro país no existe ningún estudio que correlacione dichos datos.

Este estudio quiere dar luces acerca de la importancia de la estandarización de las técnicas, con el fin de obtener mejores resultados para el paciente y la creación de literatura con el objetivo de incrementar la investigación en Latinoamérica y especialmente en Colombia.

\section{Conclusión}

El registro nacional de NLP es un estudio en tiempo real y de fácil acceso que permite obtener a gran escala datos relevantes de los pacientes a quienes se les realiza NLP en varias instituciones del país. Adicionalmente proporcionará bases para la creación de estudios multicéntricos y publicaciones de literatura colombiana.

\section{Responsabilidades éticas}

\section{Protección de personas y animales}

Los autores declaran que para esta investigación no se han realizado experimentos en seres humanos ni en animales.

\section{Confidencialidad de los datos}

Los autores declaran que han seguido los protocolos de su centro de trabajo sobre la publicación de datos de pacientes.

\section{Derecho a la privacidad y consentimiento informado.} Los autores declaran que en este artículo no aparecen datos de pacientes.

Conflicto de intereses

Los autores declaran no tener ningún conflicto de intereses. 


\section{Bibliografía}

1 Andonian S, Okhunov Z, Shapiro EY, Smith AD, Okeke Z. Diagnostic utility and clinical value of postpercutaneous nephrolithotomy nephrostogram. J Endourol 2010;24:1427-1430

2 Segura JW, Preminger GM, Assimos DG, Dretler SP, Kahn RI, Lingeman JE, et al. Nephrolithiasis Clinical Guidelines Panel summary report on the management of staghorn calculi. The American Urological Association Nephrolithiasis Clinical Guidelines Panel. J Urol 1994; 151:1648-1651

3 Thomas K, Smith NC, Hegarty N, Glass JM. The Guy's stone scoregrading the complexity of percutaneous nephrolithotomy procedures. Urology 2011;78:277-281. DOI 10.1016/j.urology.2010.12. 026

4 Smith A, Averch TD, Shahrour K, Opondo D, Daels FPJ, Labate G, et al. A nephrolithometric nomogram to predict treatment success of percutaneous nephrolithotomy. J Urol 2013;190:149-156

5 Opondo D, Gravas S, Joyce A, Pearle M, Matsuda T, Sun Y-H, et al. Standardization of patient outcomes reporting in percutaneous nephrolithotomy. J Endourol 2014;28:767-774

6 Okhunov Z, Friedlander JI, George AK, Duty BD, Moreira DM, Srinivasan AK, et al. S.T.O.N.E. nephrolithometry: Novel surgical classification system for kidney calculi. Urology 2013;81: $1154-1160$

7 Thomas K, Smith NC, Hegarty N, Glass JM. The Guy's stone scoreGrading the complexity of percutaneous nephrolithotomy procedures. Urology 2011;78:277-281

8 Labadie K, Okhunov Z, Akhavein A, Moreira DM, Moreno-Palacios $\mathrm{J}$, del Junco $\mathrm{M}$, et al. Evaluation and comparison of urolithiasis scoring systems in percutaneous kidney stone surgery. J Urol 2014;193:154-159

9 Mitropoulos D, Artibani W, Graefen M, Remzi M, Rouprêt M, Truss M. Reporting and grading of complications after urologic surgical procedures: An ad hoc EAU guidelines panel assessment and recommendations. Eur Urol 2012;61:341-349

10 Opondo D, Gravas S, Joyce A, Pearle M, Matsuda T, Sun Y-H, et al. Standardization of patient outcomes reporting in percutaneous nephrolithotomy. J Endourol 2014;28:767-774

11 De la Rosette JJ, Opondo D, Daels FP, Giusti G, Serrano A, Kandasami $\mathrm{SV}$, et al. Categorisation of complications and validation of the Clavien score for percutaneous nephrolithotomy. Eur Urol 2012; 62:246-255 